ARTICLE

\title{
An approach to balance problems and falls in elderly persons
}

\author{
L de Villiers, MB ChB, FCP (SA); S Z Kalula, MB ChB, FRCP, PhD
}

Division of Geriatric Medicine, Albertina and Walter Sisulu Institute of Ageing in Africa, Department of Medicine, Faculty of Health Sciences, University of Cape Town and Groote Schuur Hospital, Cape Town, South Africa

\section{Corresponding author: S Z Kalula (sebastiana.kalula@uct.ac.za)}

\begin{abstract}
Gait instability and falls are common in elderly persons and have devastating consequences, with substantial morbidity and mortality. Furthermore, they are a precipitant for functional decline, increasing frailty and institutionalisation. The rate of falls and severity of complications increase with age and frailty. A consequence of falls with or without injury is that at least a third of persons develop a fear of falling, which leads to functional decline and a progressive decline in gait. The causes of falls in elderly persons are multifactorial and include physiological changes of ageing, frailty, pathologies, and environmental and situational factors. Maintaining postural control requires a complex integration of sensory input, central processing, motor co-ordination and musculoskeletal function, which decrease with ageing. This change, combined with sarcopenia, leads to slowed and weakened postural control and muscle responses, resulting in gait instability and falls.

The assessment and management of a patient who is at risk of falls or who has fallen require a multidisciplinary approach to identify and address factors contributing to the fall. The assessment, which includes history, physical examination, and evaluation of gait, postural control and mental function, is aimed at identifying situational and associated factors surrounding a fall, intrinsic impairments in gait or pathologies that increase the risk of falls. The components of the assessment comprise a full medical evaluation for pathologies, including vision, medication review (including over-the-counter medication) with regard to polypharmacy and high-risk medications, psychogeriatric review, functional status (instrumental activities of daily living (IADLs) and activities of daily living (ADLs)), functional assessment of gait and balance, and assessment of environmental hazards in the home. Laboratory investigations are guided by clinical suspicions or diagnoses arising from the medical assessment and screening for common conditions that may increase the risk of falls.

Management and prevention of falls focus on maintaining mobility and balance, and identifying those at risk of a fall for multidisciplinary assessment and intervention. Intervention to reduce the risk of subsequent falls is targeted at modification of the contributory factors. Intervention includes management of underlying pathologies, strength and balance training by a physiotherapist, assessment and modification of environmental hazards in the home by an occupational therapist, medication review and rationalisation of high-risk medications and polypharmacy, and supplementation of vitamin $\mathrm{D}$ where indicated.
\end{abstract}

S Afr Med J 2015;105(8):695. DOI:10.7196/SAMJnew.8037

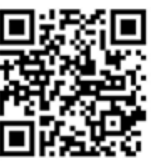

Gait instability and falls are common in elderly persons and have devastating consequences, with substantial morbidity and mortality. Furthermore, they are a precipitant for functional decline, increasing frailty and institutionalisation. ${ }^{[1]}$ The rates of falls and severity of complications increase with age and frailty. In persons $\geq 65$ years, the prevalence of falls is as high as $30 \%$ per year. ${ }^{[2]}$ Among institutionalised persons aged $>75$ years, mean fall rates are $\leq 1.7$ per bed annually, with half of affected persons having multiple falls, and $10-25 \%$ of the latter leading to fractures or lacerations requiring hospitalisation. ${ }^{[3]}$ An important consequence of falls is that at least a third of persons develop a fear of falling after the event, resulting in functional decline and a progressive decline in gait. ${ }^{[4]}$ The causes of falls in the elderly are usually multifactorial, with a combination of intrinsic factors, including the physiological changes of ageing, frailty, pathologies, and extrinsic, environmental and situational factors. In up to $10 \%$ of patients presenting with a fall the underlying cause is an acute medical condition. ${ }^{[5]}$

\section{Pathophysiology}

Maintaining postural control requires a complex integration of sensory input, central processing, motor co-ordination and musculoskeletal function to perceive environmental stimuli and respond accordingly to perturbations to control body movement (Fig. 1). The sensory information required includes vision, vestibular input and proprioception. With ageing there is a decrease in depth perception and dark-light discrimination, a high prevalence $(\leq 40 \%)$ of decreased vibration and position sense and frequent vestibular impairment. Central processing is slower, both in the interpretation and weighting of sensory information and in motor output and co-ordination. Higher-order cortical processes linking motor and sensory systems are required for planning movements, solving problems, divided attention and environmental scanning and response. ${ }^{[5]}$ Peripheral nerve conduction is slower, there is a decrease in the number of motor units in the spinal cord, and an increase in the number of myocytes with increased excitability thresholds innervated by each motor unit. ${ }^{[6]}$ This change, when combined with sarcopenia (progressive and generalised loss of skeletal muscle mass and strength), leads to slowed and weakened postural control and muscle responses. ${ }^{[7]}$ Slow postural reflexes are seen as increased postural sway (back and forth movement of head and upper trunk exacerbated by removing visual input by closing the eyes) when evaluating gait and balance. ${ }^{[6,8]}$

The assessment and management of a patient who is at risk of falls, or who has fallen, require a multidisciplinary approach to identify and address factors that contribute to a fall. Such an assessment should be considered for elderly persons who have sustained injuries after a fall, have had a single fall, have an abnormal gait, or have had $\geq 2$ unexplained falls in a 12 -month period. The history, examination and assessment of gait and postural control are aimed at identifying situational factors surrounding the falls and associated factors, 
as well as intrinsic impairments in gait or pathologies that increase the risk of falls. ${ }^{[9]}$

The evaluation of an elderly person who has fallen or has a fear of falling requires a multidimensional assessment of all factors that can contribute to the risk of falls, and is best performed by a multidisciplinary team. These components include a full medical assessment for pathologies, medication review (including over-the-counter medication) with regard to polypharmacy and high-risk medications, ${ }^{[10]}$ psychogeriatric review, functional status, functional assessment of gait and balance and an evaluation of environmental hazards in the home. The interventions to reduce the risk of subsequent falls are targeted at modifying the contributory factors. ${ }^{[9,11]}$

\section{Medical history, examination and investigation}

The history of previous falls should include the frequency, environmental factors such as time, location, floor surface, lighting, obstacles and precipitants, as well as associated factors such as footwear, use of walking aids, glasses, and substances or medications (Table 1). The systemic enquiry and physical examination are aimed at identifying causative or contributory cardiovascular, neurological, sensory, musculoskeletal or other impairments and pathologies. The value of documenting the functional status of a patient with falls is that functional impairments should be accounted for by cognitive, psychiatric and physical pathologies. ${ }^{[9,11]}$ Impairments identified in the history, examination and investigation of a patient and the success of interventions to treat and manage these problems can be gauged by improvements in functional status. Functional status is measured in terms of instrumental activities of daily living (IADLs) (activities required for independent living in the community, such as housekeeping, cooking, shopping, use of transportation, management of finances, and maintaining hobbies and social contact) and activities of daily living (ADLs) (activities that enable a person to care for him/herself, such as bathing, grooming, dressing, toileting, continence and mobility on a level surface and stairs, and ability to transfer from bed to chair) (Fig. 2).

The physical examination should include visual acuity assessment, supine and erect blood pressure, an ECG and a chest radiograph. The physical assessment focuses on the identification of cardiovascular, vestibular and central nervous system causes of dizziness/vertigo, identification of visual,

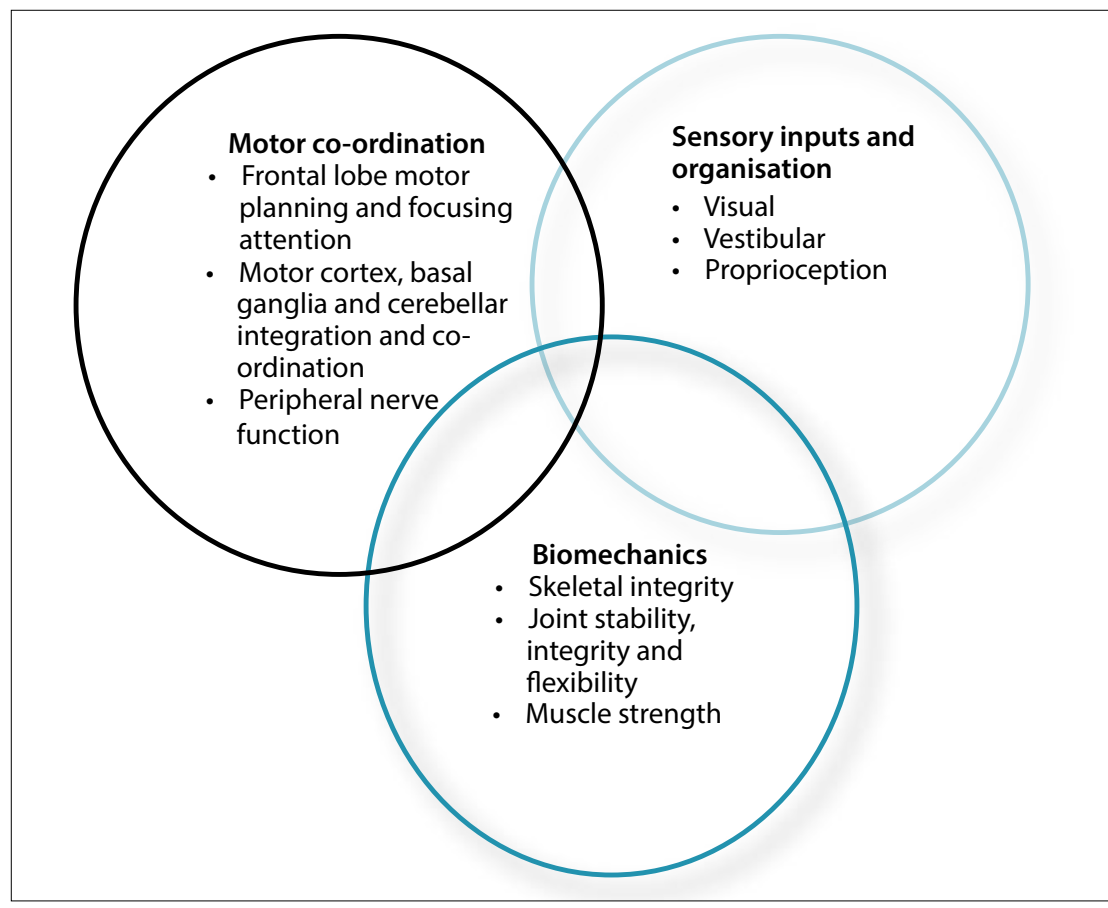

Fig. 1. Maintaining postural stability.

Table 1. Medical history and examination: symptoms, signs and possible associated pathology

\begin{tabular}{|c|c|}
\hline Symptoms & Pathologies to consider \\
\hline Light-headed on standing & Postural hypotension \\
\hline Light-headed with head turning & Carotid sinus hypersensitivity \\
\hline Chest pain/dyspnoea/palpitations & $\begin{array}{l}\text { Congestive cardiac failure, arrhythmia, } \\
\text { aortic stenosis }\end{array}$ \\
\hline Drop attacks & Vertebrobasilar insufficiency \\
\hline Dizziness/vertigo & Medication side-effects \\
\hline Brief vertigo & Benign paroxysmal positional vertigo \\
\hline Episodic vertigo and tinnitus & Ménière's disease \\
\hline Back pain relieved by flexion & Lumbar spinal stenosis \\
\hline Acute confusion & Delirium \\
\hline \multicolumn{2}{|l|}{ Signs } \\
\hline Asymmetrical spasticity & Cerebrovascular disease \\
\hline Hyperreflexia, instability & Cervical myelopathy, vitamin $B_{12}$ deficiency \\
\hline Bradykinesia, rigidity, tremor & Idiopathic Parkinson's disease \\
\hline Dementia & Alzheimer's disease \\
\hline - Plus: hyperreflexia/pseudobulbar palsy & Vascular dementia \\
\hline - Plus: parkinsonism and visual hallucination & Lewy body dementia \\
\hline - Plus: incontinence and abnormal gait & Normal pressure hydrocephalus \\
\hline Proximal myopathy & $\begin{array}{l}\text { Hypothyroidism, polymyalgia rheumatica, } \\
\text { statins, dermatomyositis }\end{array}$ \\
\hline Peripheral sensory loss & Peripheral neuropathy \\
\hline
\end{tabular}

proprioceptive and peripheral nervous system sensory input impairments, central nervous system motor, extrapyramidal and cerebellar pathology and musculoskeletal problems. Attention should specifically be paid to identifying proximal muscle weakness, as core stability is important in maintaining postural stability, especially in the event of external stress such as stumbling. Core muscle weakness is a common consequence 


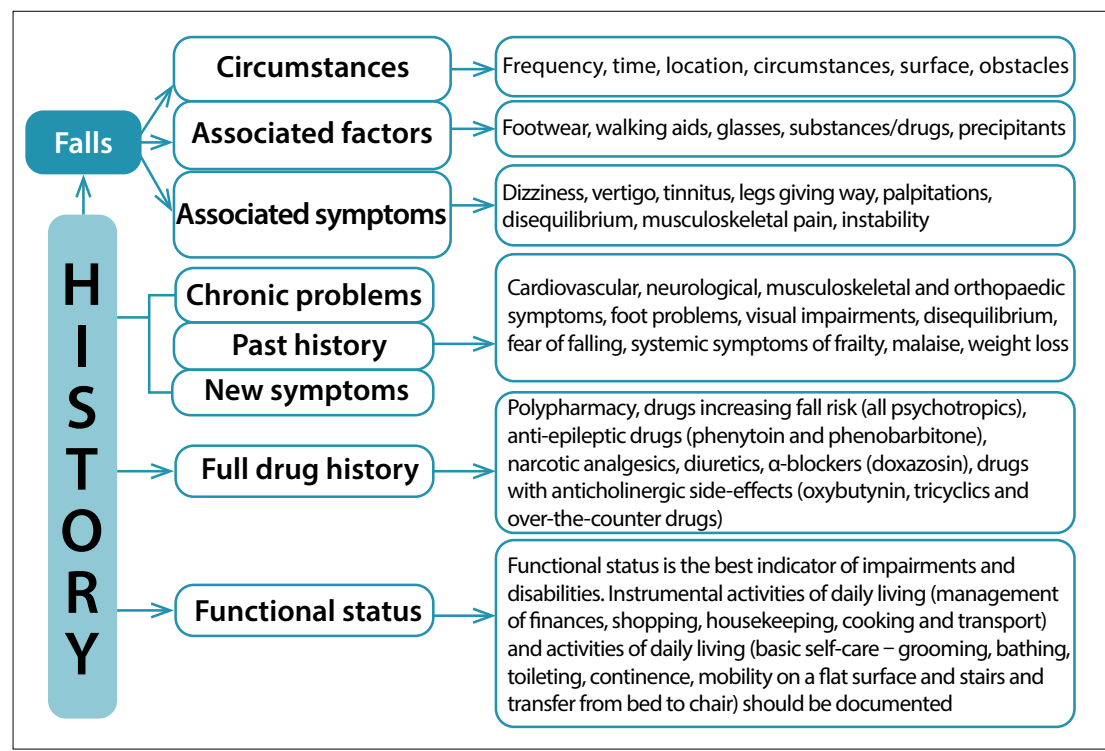

Fig. 2. Relevant history in fall assessment.

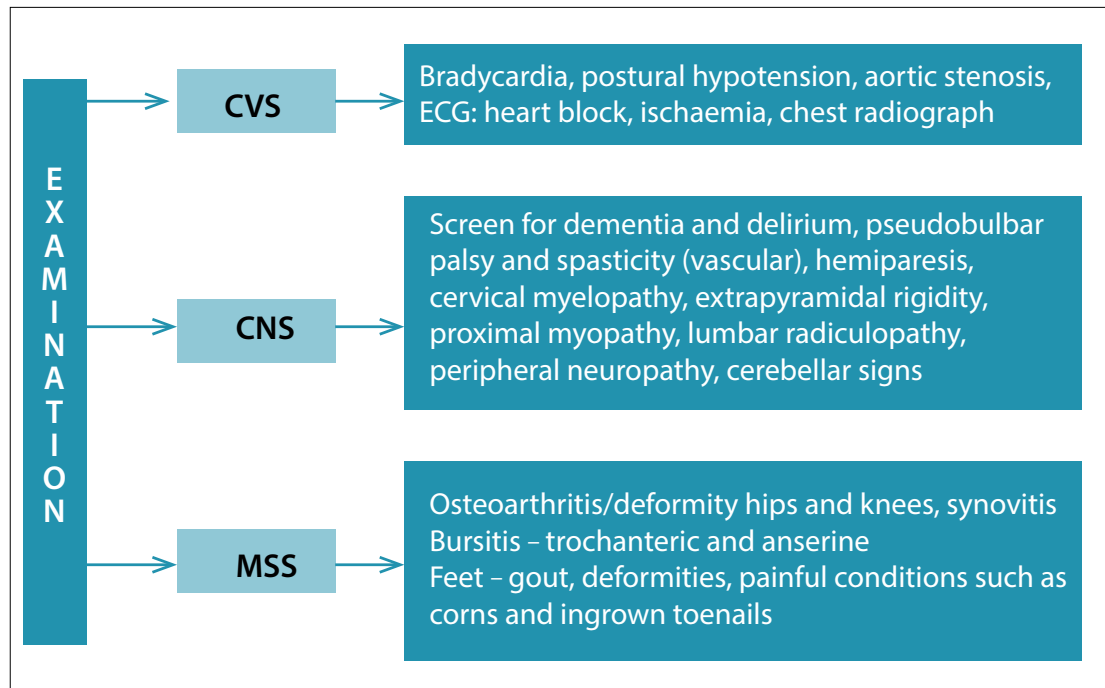

Fig. 3. Relevant physical examination $(C V S=$ cardiovascular system; $C N S=$ central nervous system; MSS = musculoskeletal system .

Table 2. Routine investigations

\begin{tabular}{|c|c|}
\hline Investigation & Condition \\
\hline Electrolytes and renal function & Hyponatraemia, hypokalaemia, renal impairment \\
\hline Calcium & Primary hypoparathyroidism \\
\hline Full blood count and white blood cell differential & $\begin{array}{l}\text { Anaemia, chronic lymphocytic leukaemia, } \\
\text { myelofibrosis }\end{array}$ \\
\hline Erythrocyte sedimentation rate & $\begin{array}{l}\text { Polymyalgia rheumatica, myeloma, tuberculosis, } \\
\text { malignancy, rheumatoid arthritis, vasculitis } \\
\text { (neuropathy) }\end{array}$ \\
\hline Thyroid-stimulating hormone & Hypothyroid proximal myopathy \\
\hline Vitamin $B_{12}$ & $\begin{array}{l}\text { Deficiency with central nervous system and } \\
\text { peripheral nerve dysfunction }\end{array}$ \\
\hline Syphilis serology & $\begin{array}{l}\text { Neurosyphilis, central nervous system and } \\
\text { peripheral nerve pathology }\end{array}$ \\
\hline Vitamin D levels & Deficiency - increased risk of falls \\
\hline
\end{tabular}

of deconditioning and frailty. If there is proximal muscle weakness in the hips from sitting to standing position in the assessment of gait and balance, shoulder muscle strength on shoulder abduction should be assessed. If there is proximal muscle weakness of both upper and lower limbs, the patient needs to be evaluated for causes of myopathy. Degenerative joint disease and osteoarthirits of the hips and knees are common in the elderly, as are foot problems such as corns, bunions and ingrown toenails. ${ }^{[1]}$ Pain and deformity result in an abnormal gait, which increases the risk of falls, especially if there are multiple contributing factors. In the event of abnormal findings on the musculoskeletal examination, osteoarthritis, gout and rheumatoid arthritis should be considered (Fig. 3).

\section{Cognitive and}

\section{psychiatric evaluation}

Cognitive assessment should be done to identify cognitive deficits of dementia or delirium, and to enquire about symptoms of depression and sleep disturbance and the presence of substance or alcohol abuse, all of which increase the risk of falls. There are numerous formal, validated screening instruments for cognitive and psychiatric disorders. A problem with the Mini-Mental State Examination (MMSE) is that it does not evaluate executive (frontal lobe) function, unlike the Montreal Cognitive Assessment (MOCA), ${ }^{[12]}$ the latter therefore being a better screening tool for the busy clinician. The recognition of delirium rests on the recognition of the fluctuating character of this disorder in terms of concentration and arousal, presence of inattention (poor concentration/ distractibility), disorientation for time and place and inability to interpret sensory stimuli (such as if someone in a nurse's uniform is at the bedside, the person believes he/she is in a hospital). Major depressive disorder is characterised by the presence of vegetative features such as sleep and appetite disturbances, low energy levels, diurnal mood disturbance, nihilistic thoughts, suicidal ideation and pervasive sadness.

Laboratory investigations include those guided by clinical suspicions or diagnoses arising from the medical assessment and screening for common conditions that may increase the risk of falls ${ }^{[11]}$ (Table 2).

\section{Gait and balance assessment}

Gait and balance are assessed to determine whether there is a balance problem, predict the risk of falls from the impairments identified, and establish the underlying 


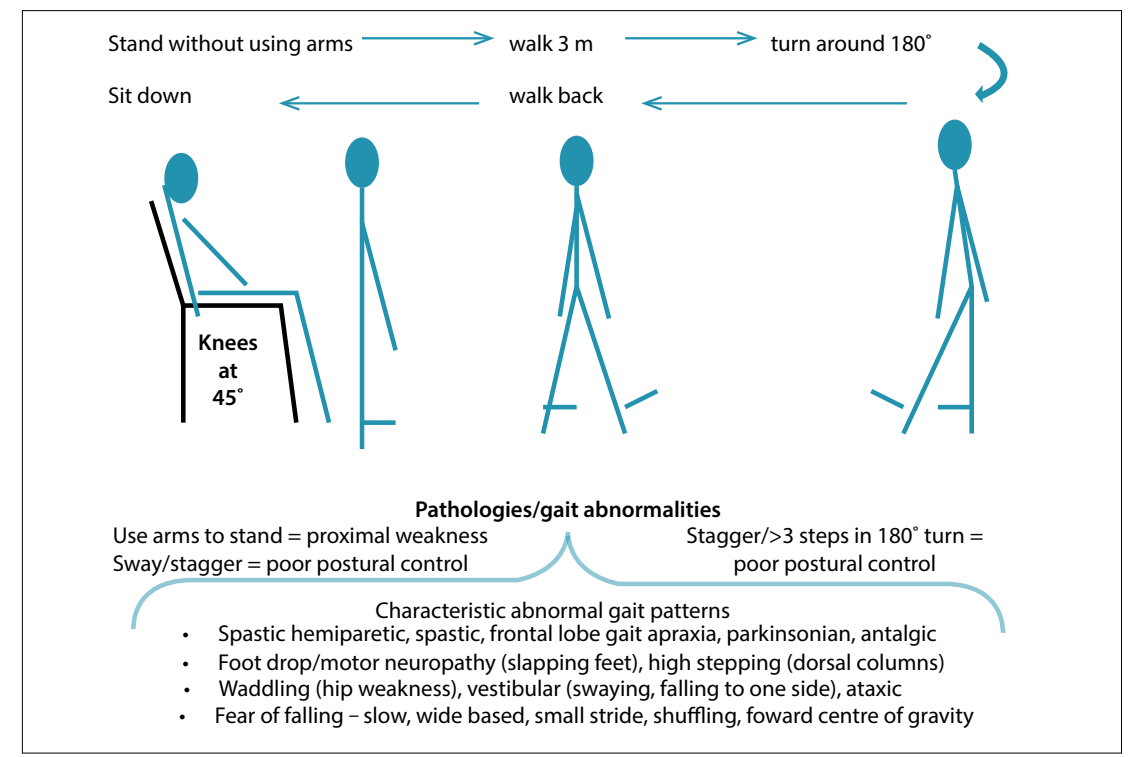

Fig. 4. The Get Up and Go test.

\section{Table 3. System components for balance assessment}

\begin{tabular}{ll}
\hline System components & Balance assessment \\
\hline Biomechanical & - Strength \\
& - Range of motion \\
& - Stiffness/compliance \\
Motor & - Mlignment \\
co-ordination & Maintain stability with postural perturbations (external forces, \\
& - Co-ordination strategies for recovering stability (latency, \\
& - sequence, scaling on sensory information) \\
& - initial conditions) \\
- Adaption of postural strategy (immediate, with experience, & long-term motor learning) \\
Sensory & - Stability in altered sensory conditions (visual/surface) \\
organisation & - Limits of stability \\
& - Perception of vertical orientation \\
& - Attention to and suppression of sensory cues
\end{tabular}

causes of the balance problem so that effective intervention may be offered. The value of functional assessment of gait and balance is that, by direct observation, one can assess the impact that physiological changes of ageing and pathologies identified in the history and examination have on gait and postural control, and subsequently on the risk of falls. The assessment of balance should include the identification of impairments between the different types of balance control, including ability to respond to external perturbations (forces), anticipation of postural responses necessitated by voluntary motions, and effective and safe movement (controlling and moving the centre of the body mass over the base of support when moving through space). If the gait and balance are normal and there are recurrent falls, the underlying problem is most likely cardiovascular or related to medications or substances.

There are three approaches to the assessment of balance: functional assessment, a systems approach and quantitative posturography. Functional assessment seeks to establish whether there is a balance problem and predict the risk of falls, while the systems approach tries to identify which of the elements of postural control are impaired. Functional assessment tools seek to rate performance across various tasks requiring balance control to identify functional limitations or the capacity to perform various tasks. This information can then be used to target medical and physical interventions at the impaired components. ${ }^{[8]}$ There are several validated tools such as the Tinetti Performance-Orientated Assessment of Mobility, the Berg Functional Balance Scale ${ }^{[1,14]}$ and the Timed Get Up and Go test ${ }^{[15]}$ (Fig. 4).

The systems approach to the assessment of balance seeks to identify impairments in the various subcomponents of postural control and compensatory strategies used to compensate for these impairments. The components assessed are described in Table 3. Compensatory strategies commonly employed by elderly persons with impaired and slowed postural control include excessive trunk/hip motion to maintain postural equilibrium, actively locking the head to the trunk to compensate for dizziness, compensating for muscle weakness by locking the knee and forward positioning of the centre of muscle mass, and widening the base of the gait and shortening the stride length to compensate for a feeling of disequilibrium.

A combination of elements of the various functional balance assessment tools suitable for a busy clinician would include the Timed Get Up and Go test, Romberg's test (identifies vestibular and proprioception impairments by removing visual compensation), assessing for postural sway with eyes open and closed (slowed postural reflexes), the sternal nudge (postural response to external force) and the functional reach test.

\section{Management of persons at risk of falls and those with fear of falling}

Principles of managing elderly persons at risk of falls, those who have fallen and those with a fear of falling are similar. Prevention of falls focuses on maintaining mobility and balance in functional, independent communitydwelling elderly persons and identifying those at risk of falls for multidisciplinary assessment and intervention. ${ }^{[16,17]}$

In independent community-dwelling elderly persons without impairments in gait and balance, the focus is on maintaining physical function, optimally managing chronic diseases, screening, ensuring good nutrition and avoiding prescription of drugs that increase the risk of falls, if possible. Exercise recommendations for maintaining physical function include 30 minutes of aerobic activity 3 times a week (e.g. walking at a brisk pace) and static strength and balance exercises such as callanetics and Tai Chi. Screening for vitamin $\mathrm{D}$ deficiency is expensive and the prevalence of suboptimal levels is high, even in South Africa, especially in the winter months. Some health professionals advocate that supplementation for all the elderly is more cost effective. 
In elderly persons with impaired gait and balance that puts them at risk of falls, a multidisciplinary intervention programme is required. Interventions that are effective in reducing subsequent falls, hospitalisation and functional decline include strength and balance training by a physiotherapist, assessment and modification of environmental hazards in the home by an occupational therapist, medication review and rationalisation of high-risk medications and polypharmacy, and the supplementation of vitamin $\mathrm{D}$ if indicated. ${ }^{[16,18]}$

\section{References}

1. Tinetti ME. Clinical practice: Preventing falls in elderly persons. N Engl J Med 2003:348(1):42-49.

2. Hausdorff JM, Rios DA, Edelberg HK. Gait variability and fall risk in community-living older adults A 1-year prospective study. Arch Phys Med Rehabil 2001;82(8):1050-1056

3. Rubenstein LZ. Falls in older people: Epidemiology, risk factors and strategies for prevention. Age Ageing 2006;35(Suppl 2):ii37-ii41.

4. Zijlstra GA, van Haastregt JC, van Eijk JT, van Rossum E, Stalenhoef PA, Kempen GI. Prevalence and correlates of fear of falling, and associated avoidance of activity in the general population of community-living older people. Age Ageing 2007;36(3):304-309.

5. Ambrose AF, Hausdorff JM. Risk factors for falls among older adults: A review of the literature. Maturitas 2013;75(1):51-61. [http://dx.doi.org/10.1016/j.maturitas.2013.02.009]
6. Horak FB. Postural orientation and equilibrium: What do we need to know about neural control of balance to prevent falls? Age Ageing 2006; 35(Suppl 2):ii7-iil1.

7. Manini TM, Clark BC. Dynapenia and aging: An update. J Gerontol A Biol Sci Med Sci 2012;67(1):2840. [http://dx.doi.org/10.1093/gerona/glr010]

8. Lord SR, Menz HB, Tiedemann A. A physiological profile approach to falls risk assessment and prevention. Phys Ther 2003;3:237-252.

9. Salzman B. Gait and balance disorders in older adults. Am Fam Physician 2010;82(1):61-68.

10. Woolcott JC, Richardson KJ, Wiens MO, et al. Meta-analysis of the impact of 9 medication classes on falls in elderly persons. Arch Intern Med 2009;169(21):1952-1960.

11. Moylan KC, Binder EF. Falls in older adults: Risk assessment, management and prevention. Am J Med 2007;120:493-497.

12. Dag E, Örnek N, Örnek K, Günay F, Türkel Y. Mini mental state exam versus Montreal cognitive assessment in patients with age-related macular degeneration. Eur Rev Med Pharmacol Sci 2014;18(20):3025-3028.

13. Bogle-Thorbahn LD, Newton RA. Use of the Berg Balance Test to predict falls in elderly persons. Phys Ther 1996;76(6):576-583.

14. Whitney SL, Poole JL, Cass SP. A review of balance instruments for older adults. Am J Occup Ther 1998;52(8):666-671.

5. Shumway-Cook A, Brauer S, Woollocott M. Predicting the probability of falls in community-dwelling older adults using the Timed Get Up \& Go test. Phys Ther 2000;80:896-903.

16. Karlsson MK, Vonschewelov T, Karlsson C, Coster M, Rosengen BE. Prevention of falls in the elderly: A review. Scand J Public Health 2013;41:442-454

17. Chang JT, Morton SC, Rubenstein LZ, et al. Interventions for the prevention of falls in older adults: Systematic review and meta-analysis of randomized clinical trials. BMJ 2004;328:676-679.

18. Murad MH, Elamin KB, Abu-Elnor NO, et al. The effects of vitamin D on falls: A systematic review and meta-analysis. J Clin Endocrinol Metab 2011;96(10):2997-3006. [http://dx.doi.org/10.1210/ jc.2011-1193 